\title{
ON A THEOREM OF HERSTEIN
}

\section{EUGENE SCHENKMAN ${ }^{1}$}

Let $A$ be a simple ring which is not a 4 -dimensional algebra over a field of characteristic 2 , and let $U$ be a Lie ideal of $A$. Then Herstein proves in [2] that either $U$ is contained in the center $Z$ of $A$, or $U$ contains $[A, A]$; if in addition $U$ is also assumed to be an associative ring and contains $[A, A]$, then $U=A$.

Herstein and Baxter also prove in $[1 ; 3]$, and $[4]$ that $[A, A]$ $\bmod [A, A] \cap Z$ is a simple Lie ring. Our object here is to give a simple proof of this fact, basing our argument only on the results of [2] quoted above, though many of Herstein's ideas will be incorporated here without explicit mention.

We first recall a few definitions and make some preliminary remarks. If $u$ and $a$ are elements of the ring $A,[u, a]$ will designate $u a-a u$, and $[U, A]$ will denote the module generated by all $[u, a]$ where $u$ is in $U$ and $a$ in $A$. $U$ is an ideal of $A$ if $U$ is a module and if $[U, A]$ is contained in $U$. If $U$ and $V$ are submodules of $A$ then $U+V$ is the module generated by $U$ and $V$. If $U$ is a submodule of $A$ we shall define $U: A$ to be the set of elements $x$ of $A$ such that $[x, A] \subset U$, thus $[U: A, A]$ is contained in $U$. It is easy to check that $U: A$ is a ring from the identity $[u v, a]=[u, v a]+[v, a u]$. When $U$ is a Lie ideal of $A$ then $U: A$ is also such.

The following identities will be needed in the sequel; they are easy to verify:

$$
\begin{aligned}
{[u, v] a } & =[u, v a]-v[u, a\rfloor, \\
b[u, v] a & =[u, v] a b+[b,[u, v] a] .
\end{aligned}
$$

We note the following facts about $U$ when $U$ is a Lie ideal of $[A, A]$; we shall let $S$ stand for $U: A$ whence $[S, A] \subset U$ and let $T=[S, S]$.

(1) If $U=U_{0}$, and for $n$ a natural number, $U_{n}$ is defined to be $U_{n-1}+\left[U_{n-1}, A\right]$ then $U_{n}$ is a Lie ideal of $[A, A]$.

(2) If $V=\sum_{n=1}^{\infty} U_{n}$ then $V$ is a Lie ideal of $A$.

(3) $U_{n+1}: A \supset U_{n}$ and $U_{n+1}: A \supset U_{n}: A$.

(4) $[U, U] \subset S$; for $[[U, U] A]$ is contained in $[[U, A] U]$ by the Jacobi identity.

(5) $[U, S] \subset S$; for $[[U, S] A] \subset[[U, A] S]+[[S, A] U]$.

(6) $[[A, S] S] \subset S$. This follows from (5) and the definition of $S$.

Received by the editors July 10, 1958.

1 The author is indebted to Professor L. I. Wade for some helpful remarks during the preparation of this paper, and to the National Science Foundation for support. 
(7) $[[S, S] A] \subset S$. This follows from (6) and the Jacobi identity.

(8) $[T, T] A \subset S$ and $A[T, T] \subset S$. The first statement follows from $\left({ }^{*}\right)$ and (7) with $u$ and $v$ in $T=[S, S]$ and $a$ arbitrary in $A$. The second statement requires a similar argument.

(9) $A[T, T] A \subset S+U$. This follows from (**) and (8) with $u, v$ in $T$, and $a, b$ arbitrary in $A$.

(10) $[S+U, S+U] \subset S$ because of (4) and (5).

These preliminaries are sufficient to prove the theorem.

Theorem. If $A$ is a non-Abelian simple ring which is not a 4 dimensional algebra over a field of characteristic 2 and if $U$ is a proper Lie ideal of $[A, A]$ then $U$ is contained in $Z$, the center of $A$.

Proof. If $S=U: A$ then $S$ is properly contained in $A$; for $[S, A]$ $\subset U$ and by hypothesis $U$ is a proper Lie ideal of $[A, A]$. If $T$ designates $[S, S]$ again, then the ideal of $A$ generated by $[T, T]$ is contained in $S+U$ by (8) and (9). If $S+U$ were equal to $A$, then by (10) $S$ would be a Lie ideal of $A$ as well as a subring; and hence by the result of [2] quoted at the outset $S$ would be in $Z$, whence $U$ would be a Lie ideal of $A=S+U$, and hence $U$ would be in $Z$ as the theorem asserts.

If now $U$ were assumed not to be in $Z$, then $S+U$ is properly contained in $A$, whence $[T, T]=0$ since $A$ is simple. This means that $[S, S]$ is Abelian. Since $U$ is not in $Z, U_{n}$ as defined in (1) is not in $Z$ for all $n$ and if $S_{n}$ denotes $U_{n}: A$ then by the above argument $\left[S_{n}, S_{n}\right]$ is Abelian. But $V$ as defined in (2) is a Lie ideal of $A$ not contained in $Z$ and hence $V=[A, A]$. We note from (3) that $S_{n} \subset S_{n+1}$ and let $Q$ denote $\sum_{n=1}^{\infty} S_{n}$. Then $Q$ is a ring which is also a Lie ideal since $Q$ contains $[A, A]$ by (3) and (2). Hence $Q=A$ by [2]. But $[Q, Q]$ is Abelian since $\left[S_{n}, S_{n}\right]$ is, and hence $[A, A]$ is Abelian.

Since $[A, A]$ is Abelian the centralizer of $A$ is a ring which contains $[A, A]$ and consequently is a Lie ideal of $[A, A]$. Hence the centralizer of $[A, A]$ is all of $A$ and $[A, A] \subset Z$. Now if $x$ and $y$ are in $A$, then $0=[[x, y x] y]=[[x, y] x, y]=[x, y]^{2}$ where $[x, y]$ is in $Z$. But then $[x, y] A$ is an ideal of $A$ not equal to $A$ since $A$ is non-Abelian and $[x, y] A$ is a zero ring. It follows that $[x, y] A=0$ and $([x, y])$ is an ideal of $A$. Again we must conclude $[x, y]=0$ and hence $A$ is Abelian contrary to hypothesis. Our assumption that $U$ is not in $Z$ leads to a contradiction and the theorem is proved.

Remark. If $U$ is a subring and also a Lie ideal of a ring $A$ then the ideal generated by $[U, U]$ is contained in $U$. For if $u$ and $v$ are in $U$ and $a, b$ in $A$ then it follows from $\left(^{*}\right)$ that $[U, U] A$ is contained in $U$ and similarly $A[U, U]$ is in $U$; furthermore from $\left(^{* *}\right)$ it follows that $A[U, U] A$ is in $U$. 


\section{BiBLIOGRAPHY}

1. Willard E. Baxter, Lie simplicity of a special class of associative ring, Proc. Amer. Math. Soc. vol. 7 (1956) pp. 855-863.

2. I. N. Herstein, On the Lie and Jordan rings of a simple associative ring, Amer. J. Nath. vol. 77 (1955) pp. 279-285. 575 .

3. - - On the Lie ring of a division ring, Ann. of Math. vol. 60 (1954) pp. 571-

4. - The Lie ring of a simple associative ring, Duke Math. J. vol. 22 (1955) pp. $471-476$.

Louisiana State University

\section{RATIONAL APPROXIMATION TO SOLUTIONS OF ALGEBRAIC DIFFERENTIAL EQUATIONS}

E. R. KOLCHIN ${ }^{1}$

Introduction. It was observed by Liouville (C. R. Acad. Sci. Paris, vol. 18 (1844) pp. 910-911; J. Math. Pures Appl. vol. 16 (1851) pp. 133-142) that an element $\alpha$ of the field $C$ of complex numbers which is algebraic of degree $n$ (over the ring $\boldsymbol{Z}$ of rational integers) can not be approximated very well by rational numbers, in the following sense: there exists a real number $\gamma>0$ such that $|\alpha-p / q| \geqq \gamma /|q|^{n}$ for all $p, q \in \boldsymbol{Z}$ with $q \neq 0$ and $p / q \neq \alpha$. Using this theorem Liouville gave the first examples of transcendental numbers. The proof depends only on the circumstance that every nonzero element of $\boldsymbol{Z}$ has absolute value $\geqq 1$ and the following two obvious facts (in the statement of which $f$ denotes the polynomial of degree $n$ vanishing at $\alpha$ ): (i) $\alpha$ is an isolated point of the set of zeros of $f$; (ii) $f(y / z)$ can be written as a fraction in which the numerator is a polynomial in $y$ and $z$ and the denominator is $z^{n}$. It follows that Liouville's theorem has an abstract version in which $\boldsymbol{C}$ and $\boldsymbol{Z}$ are replaced by an arbitrary nontrivially valued field and a nonzero subring thereof in which each nonzero element has value $\geqq 1$. Since the field $K\left(\left(X^{-1}\right)\right)$ of power series in the reciprocal of an indeterminate $X$ over a given commutative field $K$ admits a valuation for which the series $u=c_{m} X^{-m}$ $+c_{m+1} X^{-(m+1)}+\cdots\left(\right.$ with $\left.c_{m} \neq 0\right)$ has the value $|u|=e^{-m}$, and the polynomial ring $K[X]$ is a subring of $K\left(\left(X^{-1}\right)\right)$ in which every nonzero element has value $\geqq 1$, Liouville's theorem applies in this situa-

Received by the editors July 25, 1958.

1 This paper was prepared in connection with a grant from the National Science Foundation. 